
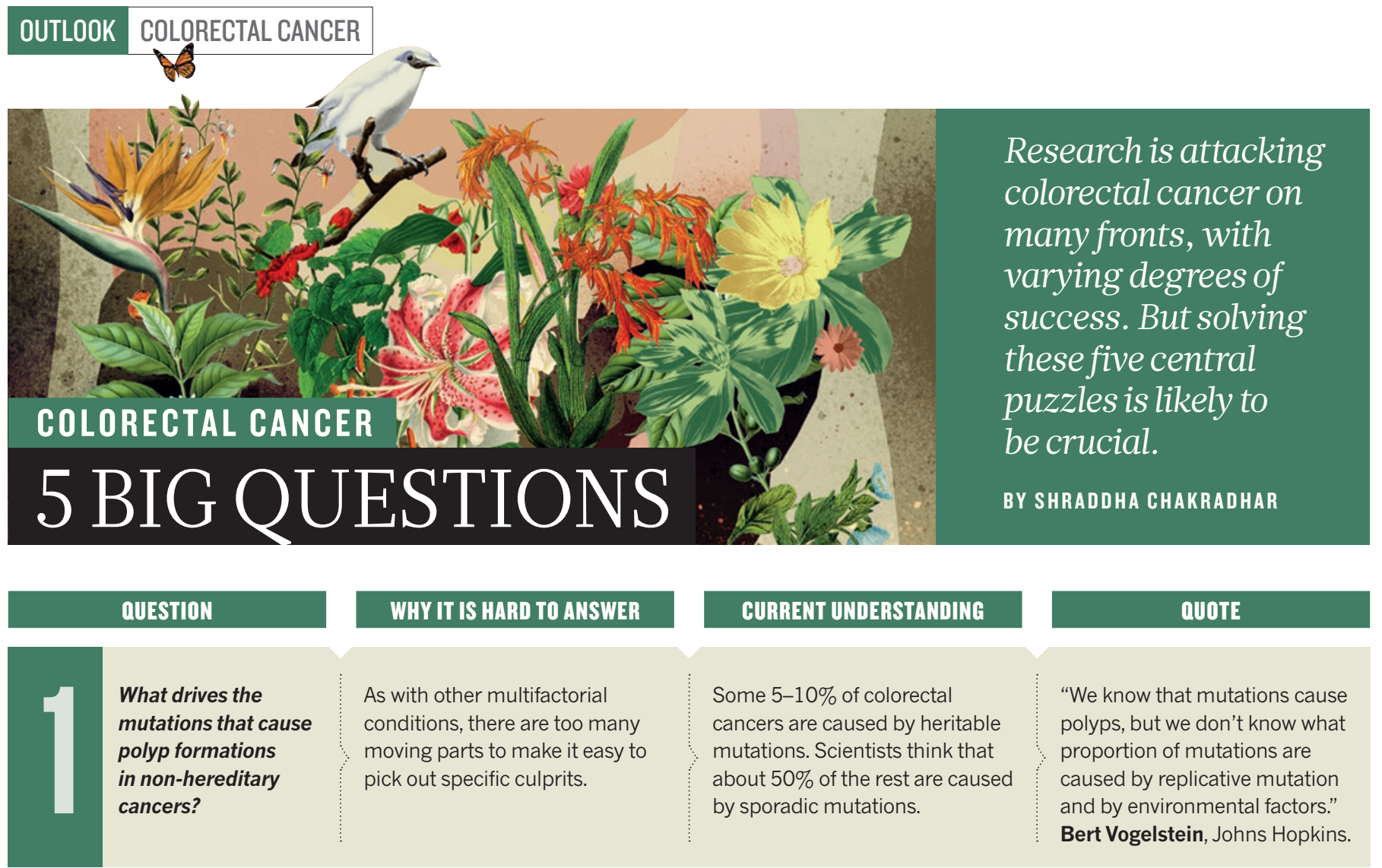

QUESTION

What drives the

mutations that cause

polyp formations

in non-hereditary

cancers?

\section{WHY IT IS HARD TO ANSWER}

As with other multifactorial conditions, there are too many moving parts to make it easy to pick out specific culprits.
CURRENT UNDERSTANDING

Some $5-10 \%$ of colorectal cancers are caused by heritable mutations. Scientists think that about $50 \%$ of the rest are caused by sporadic mutations.

\section{QUOTE}

"We know that mutations cause polyps, but we don't know what proportion of mutations are caused by replicative mutation and by environmental factors." Bert Vogelstein, Johns Hopkins.

\section{What determines whether a polyp will become cancerous?}

Researchers must tease out the sequence of mutation events that drives this transformation.
Larger polyps are more likely to become cancerous. Adenomas, one of the main types of polyp, lead to $95 \%$ of all colorectal cancers, but fewer than $10 \%$ of adenomas become cancerous.

Sedentary lifestyles and diets that are high in fat and red meat are associated with an increased risk.
"A lot of the information we have is from [people with] genetic abnormalities, and that only helps us infer what happens in normal people." Neil Hyman, University of Chicago Medical School.
What environmental factors contribute to the progression of disease?
Isolating crucial factors and combinations is difficult. Clinical trials take many years.
"All the empirical studies that have tried to determine what about the diet causes cancer haven't really been that successful." Heidi Nelson, Mayo Clinic.

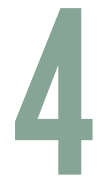

\section{What makes some} cancers more responsive to certain therapies than others?

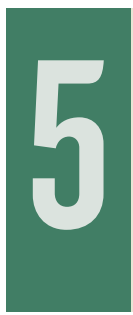

\section{How can}

immunotherapy be harnessed to treat colorectal cancers?
Colorectal cancer shows a high degree of heterogeneity in mutations, both between and within individual tumours.
Precise molecular profiles of tumours will help to predict the response to targeted therapies or combinations.
"We're throwing the dice - we really don't know what's going to work and what isn't."

Vogelstein.

Shraddha Chakradhar is a news editor at Nature Medicine.

Some tumours have infiltrating lymphocytes, which are white blood cells that can aid in killing the tumour.
"Every patient who shows up at my door wants to know if we can activate their immune system to kill cancer." Charles Fuchs, Dana-Farber Cancer Institute. immunotherapies are in trials, but any clinical impact is years away.

We lack good ways of studying colorectal cancer in humans, and animal models (mostly mice) are inadequate. Some 